\title{
Experimental investigation and model validation of the shear strength of hybrid interfaces up to complete failure
}

D. Castagnetti ${ }^{1}$, E. Dragoni ${ }^{1}$

${ }^{1}$ Univ. of Modena and Reggio Emilia, Via G. Amendola 2, 42122 Reggio Emilia, Italy.

Phone: 00390522522634

Fax : 00390522522609

E-mail: davide.castagnetti@unimore.it

\section{Abstract}

11 The paper experimentally investigates hybrid interfaces pressure-reinforced and bonded with anaerobic

12 adhesive. While their static strength has been deeply investigated, their behavior up to complete failure

13 lacks of a constitutive model. This work aims to assess the applicability of a simple model involving a

14 cohesive law and a pure friction law, in order to describe the interface behavior up to complete failure

15 under different contact pressure levels. A systematic experimental campaign investigates the shear 16 strength of cylindrical specimens butt-bonded and pressure reinforced over an annular surface. The tests

17 involve two anaerobic adhesives and four pressure levels. The experimental torque-rotation curves 18 confirm that the strain energy up to complete failure is given by a cohesive term and a pure friction term,

19 both of them linearly dependent upon the contact pressure.

21 Keywords: anaerobic, analytical models, joint design, interference fit, hybrid interfaces, cohesive-

22 friction model, shear failure. 


\section{1. Introduction}

2 The work experimentally investigates the shear strength of hybrid interfaces, under different contact

3 pressure levels. The peculiar feature of these hybrid interfaces is the combination of interference fit to

4 bonding with anaerobic adhesives.

5 The work aims to assess if the combination of a cohesive law with a pure friction law is able to describe

6 the response of an hybrid interface up to complete failure: in particular, to put to test a simple response

7 linearly dependent by the contact pressure, summing up to a constant term. Anaerobic adhesives are a

8 simple and effective solution to improve the performances of friction joints, where the coupling forces

9 are provided by mechanical clamping ${ }^{1-6}$. According to the literature ${ }^{7-10}$, the static shear strength of

10 hybrid friction bonded joints is the result of two contributions: the strength of the adhesive and the

11 friction between the interfaces of the joint. However, the experimental results from Dragoni and Mauri

$12{ }^{11}$ show that the shear strength of hybrid joints increases with contact pressure, but, for the same joint,

13 the increase rate strongly depends on the type of anaerobic adhesive.

14 Figure 1 (Dragoni and Mauri ${ }^{12}$ ) shows the experimental static strength in cylindrical fits under different

15 tightening forces and different interface conditions. The diagram shows that a strong anaerobic adhesive

16 always increases the strength of the joint compared to that of the dry interface. By contrast, a weak

17 anaerobic adhesive increases the joint strength, compared to that of the dry interface, only below a given

18 tightening force value. According to Dragoni et al. ${ }^{11-13}$, this behavior can be explained by a simple

19 micro-mechanical model, relying on the assumption that the adhesive always forms a thin layer between

20 the adherend protrusions. According to this model, the high local pressure acting on the thin adhesive

21 layer retained between the crests of the mating surfaces, significantly improves its shear strength upon

22 the adhesive's at zero pressure. For a given local contact pressure, the stronger the adhesive the higher

23 the shear strength increase. Both a systematic experimental test plan ${ }^{14}$, and a microscopic-scale finite

24 element simulation plan ${ }^{15,16}$ confirmed the applicability of this simple model, and consequently of a

25 thin layer of anaerobic adhesive retained between the protrusions of the clashing surfaces. 
1 In addition, by examining the experimental curves ${ }^{14}$, it appears that the combination of a cohesive

2 response with a pure friction law can describe the response of the hybrid joint up to complete failure.

3 Similar problems in the technical literature apply this approach. Chaboche et al. ${ }^{17,18}$ propose two new

4 interface models able to describe the mixed mode progressive decohesion, followed by a contact/friction

5 behaviour after complete separation. Raous et al. ${ }^{19}$ present a model which includes both unilateral

6 contact, Coloumb friction and adhesion. Del Piero and Raous ${ }^{20}$ introduces a general framework for

7 models describing adhesive contact between rigid bondies. In ${ }^{21}$, Alfano and Sacco suggest a cohesive

8 friction model to describe problems where interface debonding plays a crucial role: for example in

9 composite or masonry structures. In ${ }^{22}$, the same approach is generalized to study the fracture

10 propagation in concrete constructions, by also considering the effect of water pressure on the fracture

11 surfaces. With regard to hybrid interfaces bonded with epoxy adhesives, Oinonen and Marquis propose

12 a similar approach ${ }^{23,24}$ combining a cohesive law together with a complex evolution model of the

13 interface damage. The main limit of this model is given by the number of material parameters that need

14 experimental calibration.

15 However, in the literature it is not possible to retrieve constitutive models to describe the response up to

16 complete failure of hybrid interfaces, where interference fit sums up to bonding with anaerobic adhesive.

17 This work aims to overcome this limitation by experimentally investigating the shear strength of hybrid

18 interfaces up to complete failure and evaluating if their response linearly increases with contact pressure

19 upon a constant term. The work involves two steps. The first step deals with the design, set-up, and

20 calibration of the experimental test bench, with specific focus on the measurement system. The second

21 step performs a preliminary systematic experimental test plan to investigate the response of the hybrid

22 interface up to complete shear failure. Two are the variables of the test plan: the adhesive type (a weak

23 and a strong anaerobic adhesive) and the nominal contact pressure between the adherends (over four

24 levels). 
1 The experimental results of this preliminary investigation suggest that the response of the hybrid 2 interface is dominated by two contributions: 1) a cohesive failure energy needed to collapse the thin 3 adhesive layer between the surfaces of the interface and 2) by dry friction between the clashing surfaces

4 of the hybrid interface after failure of the adhesive. The experimental results show that both these terms 5 linearly depend on the nominal contact pressure, in addition to a constant term, with the strong adhesive 6 largely over-performing. By examining the experimental curves, the authors extract some fundamental 7 parameters that, in a future development, can be used to fit a suitable constitutive model that accounts 8 for both cohesion and friction. Additional experimental tests are needed to confirm these preliminary 9 results. 


\section{2. Method}

2 Figure 2a presents the technical drawing of the tubular adherend used to build the specimen for the 3 experimental tests. Starting from a $\varnothing 22 \mathrm{H} 7$ grinded bar made of normalized mild steel (C40), the

4 adherends were manufactured on a lathe and bonded head to head on the annular surface shown in Figure

5 2b. The inner and outer diameter ( $16 \mathrm{~mm}$ and $22 \mathrm{~mm}$ respectively) of this bonded surface were designed 6 according to the maximum axial force $(25 \mathrm{kN})$ and torque $(200 \mathrm{Nm})$ that the servo hydraulic testing 7 machine (MTS MiniBionix 858, Eden Prairie, MN, USA) used for the tests can apply. Under the 8 maximum axial force, it comes a nominal contact pressure equal to about $134 \mathrm{MPa}$.

9 Since contact occurs between rough surfaces, due to the locally hydrostatic stress state originating on 10 the surface protrusions, the adherends steel locally builds up a virtual yield strength three times higher 11 than its macroscopic value. These results are in accordance with the fundamental assumptions of the 12 elementary tribological models of contacting rough solids ${ }^{25}$ and are a peculiar feature of Herzian 13 contacts: the surface elements experience compressive stresses in all the three orthogonal directions, 14 thus allowing the maximum pressure at the center of the contact zone to reach a value about three times 15 the macroscopic uniaxial tensile yield strength. It comes that the material. Thus, the contact pressure 16 between the plastically strained protrusions becomes equal to about three times the macroscopic yield 17 strength value measured experimentally. In conclusion, by applying an average contact pressure equal 18 to the macroscopic yield strength value of the material, the real contact surface becomes equal to about 19 one third the nominal bonding surface.

\section{2.1. Set-up of the test bench}

21 In order to accurately measure the rotation angle of the tubular specimen both in the elastic and in the 22 post-elastic region up to complete failure, a rotational encoder was installed on the specimen close to 23 the hybrid interface. 
1 Figure 3 shows the overall view of the specimen fixed to the crossheads of the test machine and

2 including the encoder for the angle measurement. The encoder is an incremental, hollow shaft, Hengstler

3 RI 76TD model ${ }^{26}$ : its inner diameter is equal to $30 \mathrm{~mm}$, the resolution is 10000 pulses for each rotation,

4 it needs a continuous input voltage equal to $5 \mathrm{~V}$, and the interface communication protocol is an RS 422

5 TTL with up to four output channels available (2 main channels and their inverted signals). The rotor of

6 the encoder ((7) in Figure 3) is fixed to the top adherend (1) through a centering bushing (8). In order to

7 minimize the measurement errors, the bushing was manufactured with tight tolerances both on the inner

8 and outer diameter. The encoder frame (6) is fixed to the clamp (5) through a bolt-nut fastener. Similarly,

9 the annular clamp (5) is tightened to the bottom adherend (2) through a bolt-nut fastener (9). This system

10 allows to register the rotation of the top adherend (1), which is actuated by the top crosshead (3), with

11 respect to the bottom adherend (2), which is fixed to the bottom crosshead (4). Specifically, the peculiar

12 feature of this system is the accurate measurement of the relative rotation between the adherends very

13 close to the hybrid interface, thus eliminating the compliances due both to the adherends and to the

14 whole kinematic chain of the test machine.

15 Using a sampling frequency of $1000 \mathrm{~Hz}$, a National Instruments USB6251 data acquisition system ${ }^{27}$ 16 registered on a notebook the signal provided by the encoder. The same data acquisition system got, from 17 the MTS testing machine controller, the torque measured by the load cell, in terms of an output voltage 18 in the range from $-10 \mathrm{~V}$ up to $+10 \mathrm{~V}$. Both the rotation angle and the torque signal were processed by 19 the Labview software ${ }^{28}$, which synchronized the signals and converted them in the rotation angle and 20 torque load respectively, through a purposely developed algorithm. On the one hand, the rotation angle 21 is proportional to the number of square wave pulses (up and down) of each main signal. Thanks to the $2290^{\circ}$ out of phase between the square waves, the resolution was equal to $0.009^{\circ}$. On the other hand, the 23 torque load is proportional to the output voltage. The Labview software was installed on the same 24 notebook that managed the data acquisition system. Figure 4 shows a picture of the test set-up on the 25 testing machine, including both the specimen and the angle measurement system. 


\section{2.2. Experimental test campaign}

2 Table 1 reports the two variables of the full factorial experimental test plan. The test plan involves a

3 weak anaerobic adhesive (Loctite $243^{29}$, from Henkel, Milano, Italy) and a strong anaerobic (Loctite

$4638^{30}$, from Henkel, Milano, Italy). Since polymerization starts, up to the end of the test, the hybrid

5 interface was subject to one of the following clamping pressure values: $0.5 \mathrm{MPa}$ (given by an axial load

6 of $90 \mathrm{~N}$ ), $45 \mathrm{MPa}$ (corresponding to $8.1 \mathrm{kN}$ ), $90 \mathrm{MPa}$ (equal to $16.2 \mathrm{kN}$ ), and $134 \mathrm{MPa}$ (corresponding

7 to an axial load of $24 \mathrm{kN}$ ). The lower nominal clamping pressure value gives a uniform contact on the

8 hybrid interface. Three replications were performed for each test configuration giving a total of 24 tests.

9 For a sample equal to a quarter of the adherends, the bonding surface roughness was measured through

10 an electronic rugosimeter (Hommelwerke ${ }^{31}$ ).

11 The bonded specimen preparation, polymerization and testing involved the same procedure as used in

12 14: manual abrasion through sandpaper (P120), cleaning with Loctite 7063 degreaser and roughness

13 measurement (through a Hommelwerke electronic rugosimeter (Lamone, Switzerland)) of the bonding

14 surface of the adherends. After installing the adherends on the hydraulic fixtures of the testing machine,

15 in order to verify the correct orthogonality of the bonding surfaces, we measured the contact area under

16 two load levels ( $1 \mathrm{kN}$ and $24 \mathrm{kN})$, by interposing a layer of tissue paper facing with one of carbon paper.

17 Then, the measurement system for the rotation angle (encoder on the top adherend and annular clamp

18 on the bottom adherend), was installed and the anaerobic adhesive was dispensed on the bottom

19 adherend's annular surface. After quasi static application of the axial load up to the desired

20 polymerization pressure, the encoder was connected to the annular clamp on the bottom adherend

21 through the bolt-nut fastener ((5) in Figure 3). The whole polymerization process involved a $45^{\circ} \mathrm{C}$

22 temperature on the specimen, obtained through a couple of infrared lamps illuminating the specimen

23 from opposite direction, which sums up to the heat dissipated by the hydraulic circuit of the machine.

24 The failure tests applied a relative rotation between the specimen's adherends equal to $90^{\circ}$, at a quite 25 slow rate of $0.2 \%$, by keeping the preload. Such a large rotation angle value was chosen in order to 
1 reach at the end of the test a steady torque load for all the specimens. The current experimental set-up

2 could be used in the future also to investigate any viscous or rate-dependent effects in the hybrid joint.

3 In order to perform accurate replications, the test procedure has been implemented in the control

4 software of the hydraulic test machine, and the tests were performed in a randomized order ${ }^{32}$. 


\section{3. Results}

2 Figure 5 shows the typical contact area of a specimen under the lowest ( $0.5 \mathrm{MPa})$ and highest (134 MPa) 3 clamping pressure value. The average roughness $\left(R_{a}\right)$ of the bonding surface of the adherends was equal 4 to about $1.5 \mu \mathrm{m}$ for the sample examined.

5 For the Loctite 243 anaerobic adhesive, Figure 6 displays the curves of the torque load registered on the

6 specimen as a function of the twist angle between the adherends: each diagram refers to one of the four 7 contact pressure values (from low to high clockwise) and includes a curve for each replication. With the 8 same layout, Figure 7 shows the same diagrams for the Loctite 638 adhesive.

9 Figure 8 shows the failed surfaces of the hybrid interfaces bonded with Loctite 243 (Figure 8a) and 10 Loctite 638 (Figure 8b) anaerobic adhesive; each of them includes a close-up view (100x magnification).

11 Figure 9 plots the shear strength, $\tau_{R}$, which corresponds to the shear stress at the peak of the curve, as a 12 function of the nominal contact pressure, $p$, applied on the hybrid interface. The failure shear strength, $13 \tau_{R}$, was calculated as the ratio between the failure torque $M_{t R}$, and the equivalent inertia moment, 14 according to the following formula:

$$
\tau_{R}=\frac{16 M_{t R}}{\pi\left(D^{2}-d^{2}\right)(D+d)}
$$

15 where $D$ is the outer diameter and $d$ the inner diameter of the specimen's adherend. The blue solid line 16 represents the best linear fitting of the experimental data. 


\section{4. Discussion}

2 The adherends exhibited an appropriate and uniform surface roughness value on the bonding surface.

3 Combined to the accurate manufacturing in terms of tight geometric tolerances, this feature enhanced a

4 precise specimen execution and a uniform distribution of the contact pressure on the whole annular

5 surface (Figure 5).

6 In all the test conditions and for both adhesives, the curves of the torque load as a function of twist angle

7 (Figure 6 and Figure 7) testify a good repeatability, specifically in the linear elastic response. A light

8 scatter appears in the failure torque, while the curves are quite close in sub-sequent post-elastic response,

9 showing a decrease of the torque up to a common asymptotic value. The increase of the nominal contact

10 pressure significantly improves the static strength the hybrid interface. With regard to the twist angle

11 where the torque decreases to the asymptotic value, the following observations can be made. For a 0.5

12 MPa contact pressure (Figure 6a and Figure 7a) the torque load falls to zero nearly immediately, and

13 thus the twist angle is a bit higher than zero. At higher contact pressure levels, a remarkable difference

14 between the two adhesives appears, and, despite some scatter between the replications, a unique angle

15 value can be retrieved for each adhesive. With regard to Loctite 243 , the twist angle where all the torque

16 load curves decrease to the asymptotic value is equal to about $20^{\circ}$ : see, in particular, the black curve in

17 Figure 6b, the red curve in Figure 6c, and the green curve in Figure 6d. By contrast, for the Loctite 638

18 adhesive (Figure $7 \mathrm{~b}, \mathrm{c}$, and d), the twist angle where the torque load becomes steady, is about $80^{\circ}$ : in

19 particular, in Figure $7 \mathrm{~b}$, the red curve decreases quite slowly up to about $80^{\circ}$.

20 In addition, in the case of the weak adhesive (Loctite 243, Figure 6), the torque load curves exhibit a

21 little increase in the end of the post-elastic response: this behavior can be imputed to a dry contact

22 condition between the surfaces, since the thin adhesive layer initially interposed between the surface

23 protrusions is totally worn out.

24 Figure 8 clearly shows a thin adhesive layer on the surface of both specimen adherends, thus testifying

25 that an hybrid interface was obtained, and cohesive failure occurs. 
1 Figure 9 highlights that for both adhesives the shear strength of the hybrid interface (bonded and pressure

2 reinforced) linearly increases with nominal contact pressure upon that of the adhesive at zero pressure.

3 Compared to the weak anaerobic, the strong adhesive exhibits a remarkably higher shear strength (about

4 four times) and a slightly higher shear strength rate with pressure. 


\section{5. Cohesive-friction model}

2 In addition to the shear strength, $\tau_{R}$ (Figure 9), of the hybrid interface, the preliminary experimental

3 results in Figure 6 and Figure 7 provide other useful data to implement a constitutive model able to

4 describe the hybrid interface. By examining the qualitative curve of the shear stress as a function of the

5 relative sliding between the specimen surfaces the following parameters can be calculated (Figure 10a):

6 the stiffness, $k$, of the linear elastic part of the response; shear stress increase, $\Delta \tau$; the residual shear

7 strength, $\tau_{\min }$, of the interface when complete cohesive failure occurs; the cohesive fracture energy, $G_{c}$,

8 needed to obtain a complete failure of the thin adhesive layer on the hybrid interface; the friction strain

9 energy, $G_{f}$, originated by the friction between the surfaces of the hybrid interface. For the sake of clarity,

10 Figure 10b shows the experimental shear stress as a function of the relative sliding (solid circles) for

11 Loctite 638, at a contact pressure equal to $134 \mathrm{MPa}$. In particular, the close-up view highlights the high

12 sampling ratio used in the whole test plan, which ensures the reliability of the numerical integration for

13 subsequent parameters calculation. Since the experimental curves are quite similar and were registered

14 using the same resolution, only this curve was reported.

15 Figure 11 describes, both for the Loctite 243 (Figure 11a) and for the Loctite 638 (Figure 11b) anaerobic 16 adhesive, the stiffness, $k$, as a function of the nominal contact pressure, calculated on the curves in Figure 176 and Figure 7. The parameter, $k$, was calculated on the linear elastic part of each curve, as the ratio 18 between the increase in the elastic shear stress and that of the relative sliding between the bonded 19 surfaces (see Figure 10). The diagrams report the values of the three replications for each contact 20 pressure level and the continuous line represents the best fit of the experimental data.

21 With the same layout, Figure 12 shows, for both adhesives, the shear stress increase, $\Delta \tau$, for each contact

22 pressure level. This parameter was calculated as the difference between the failure shear strength of the 23 hybrid interface, $\tau_{R}$, and the residual shear stress in the post-elastic field, $\tau_{\min }$ (see Figure 10 ). 
1 Similarly, Figure 13 describes for both adhesives the residual shear strength, $\tau_{m i n}$, registered on the

2 interface in the post-elastic response. This parameter represents the minimum shear strength of the joint 3 when complete failure occurs (see Figure 10).

4 Finally, it is possible to retrieve the energy needed up to complete failure of the hybrid interface, which 5 is the sum of two terms: the cohesive fracture energy, $G_{c}$, corresponding to the hatched area in Figure 610 , and the strain energy due to friction, $G_{f}$, corresponding to the dotted trapezoidal area in Figure 10. 7 Both these parameters were calculated through numerical integration using the trapezoidal rule, from 8 zero up to a maximum relative sliding $\Delta$, corresponding to the twist angle where the curve decreases to 9 the asymptotic value (see Section 4). Figure 14 and Figure 15 show, respectively, the cohesive fracture 10 energy, $G_{c}$, and the strain energy due to friction, $G_{f}$. Each point in the diagram refers to the average 11 value, while the vertical scatter bar extends plus and minus one standard deviation, as calculated from 12 the three replications of the tests.

13 On the whole, these diagrams show that:

14 - as conceivable (see the Introduction section), all the examined responses exhibit a linear increase 15 with contact pressure upon a constant term (see Figure 9, Figure 11- Figure 15, and Table 2); this 16 encouraging preliminary results suggest to improve the experimental investigation and to develop a 17 theoretical model to find a rationale for this behavior, for example using an energetic approach.

18 - for all the responses the strong anaerobic adhesive provides higher performances, with exception of 19 the stiffness, which features a remarkable decrease as contact pressure increases;

20 - the scatter in the responses of the strong anaerobic (Loctite 638) is higher than for the weak anaerobic 21 (Loctite 243);

22 - all the responses exhibit a linear trend as the contact pressure increases, with exception of the stiffness 23 of the strong anaerobic adhesive, which has a quadratic behavior;

24 - with regard to the increase in shear stress, $\Delta \tau$ (Figure 12), the strong anaerobic exhibits a remarkably 25 higher response than the weak adhesive at low pressure, but nearly the same at high pressure; 
1 - the residual shear strength, $\tau_{\min }$, of both the adhesives (Figure 13) starts from a value that is nearly

2 zero; the curve show a linear increase with contact pressure, with a rate for the strong anaerobic

3 adhesives equal to three times that of the weak anaerobic;

4 - despite the remarkably different values between the adhesives, the tests proved that both the cohesive

5 fracture energy, $G_{c}$, and the friction strain energy, $G_{f}$, linearly increase with contact pressure (Figure

614 and Figure 15). Specifically, with regard to the friction strain energy, $G_{f}$, this is physically quite

7 reasonable since $G_{f}$ is proportional to the minimum shear stress, $\tau_{\min n}$.

8 - the cohesive fracture energy, $G_{c}$, of the strong anaerobic is nearly five times higher than that of the

9 weak anaerobic, on the whole contact pressure range (Figure 14);

10 - similarly, the friction strain energy, $G_{f}$, of the strong anaerobic overcomes of nearly ten times that of 11 the weak anaerobic (Figure 15);

12 - compared to the friction strain energy, $G_{f}$ (Figure 14), the cohesive fracture energy, $G_{c}$ (Figure 15),

13 is nearly one order of magnitude higher, for both adhesives. This result directly comes from the

14 choice of the relative sliding distance $\delta$, and thus from the twist angle where the torque decreases to an asymptotic value, which is quite straightforward to be determined from the experimental failure curves (see Discussion section).

17 Table 2 reports the analytical relationships of the curves fitting the experimental data described in Figure

189 , and from Figure 11 up to Figure 15. These relationships are a useful reference for the design of hybrid 19 bonded joints, both in the elastic and in the post-elastic field. Bolted joints, flanged couplings, and 20 interference fits are typical applications where mechanical tightening can be effectively improved by 21 anaerobic adhesives. In particular, bolted joints and flanged couplings feature head to head cohesion 22 supported by interface contact pressure, while the torque failure mode is common to the vast majority 23 of hybrid bonded joints.

24 Future development of the work will improve this preliminary experimental campaign both by 25 increasing the number of replications and by considering more values for the clamping pressure. On the 
1 one hand, this could verify the authors' assumption of a quadratic behavior of the stiffness $k$ (see Figure

2 11). On the other hand, this could reduce the uncertainties observed for low pressure (see Figure 13). In 3 addition, different anaerobic adhesives will be examined in order to assess if their response has the same 4 trend with contact pressure. 


\section{6. Conclusions}

2 The work investigated the experimental response up to complete failure of hybrid pressure-reinforced 3 and bonded interfaces. The tests involved a simple specimen butt bonded on an annular surface, and

4 investigated two variables: the type of anaerobic adhesive (Loctite 243 and Loctite 638), and the nominal 5 contact pressure (over four levels, up to $134 \mathrm{MPa}$ ). The curves of the torque load as a function of twist 6 angle, accurately measured through an encoder on the specimen, showed a good repeatability. The 7 experimental results highlight a remarkably higher response of the strong anaerobic (Loctite 638) 8 compared to the weak (Loctite 243), both with regard to the static shear strength and to the post-elastic 9 response. In addition, these curves prove that the strain energy needed to produce complete failure of 10 the interface sums up two contributions: first, a cohesive term, and second, a friction term, both of them 11 linearly dependent upon contact pressure. 
3 1. Haviland, G. S., Machinery Adhesives for Locking, Retaining and Sealing. (CRC Press, New $4 \quad$ York, 1986). 1st ed.

2. Dragoni, E., and Mauri, P., Hybrid Adhesive Joints. da Silva, L. F. M., and Pirondi A (Eds.), (Springer Berlin Heidelberg, 2011), pp. 211-226.

9 3. Sekercioglu, T., and Meran, C., Mater. Des. 25, 171-175 (2004).

11 4. Sekercioglu, T., Gulsoz, A., and Rende, H., Mater. Des. 26, 377-381 (2005).

12

13 5. Sekercioglu, T., Int. J. Adhes. Adhes. 25, 352-357 (2005).

14

6. Aronovich, D. A., Murokh, A.F., Sineokov, A.P., et al., Polym. Sci. Ser. D 1, 260-265 (2008).

7. O’Reilly, C., SAE Intl Congress and Exposition. Detroit: SAE (1990).

8

8. Croccolo, D., De Agostinis, M., and Vincenzi, N., Int. J. Adhes. Adhes. 30, 359-366 (2010).

9. Croccolo, D., De Agostinis, M., and Vincenzi, N., Int. J. Mech. Sci. 56, 77-85 (2012).

10. Croccolo, D., De Agostinis, M., Mauri, P., et al., Int. J. Adhes. Adhes. 53, 80-88 (2014).

11. Dragoni, E., and Mauri, P., Proceedings of the I MECH E Part L Journal of Materials: Design and Applications 216, 9-15 (2002).

12. Dragoni, E., and Mauri, P., Int. J. Adhes. Adhes. 20, 315-321 (2000).

30 13. Dragoni, E., J. Adhes. 79, 729-747 (2003).

31

14. Castagnetti, D., and Dragoni, E., J. Adhes. 89 642-659 (2013).

33

34 15. Castagnetti, D., and Dragoni, E., Computational Materials Science 64, 146-150 (2012).

36 16. Castagnetti, D., and Dragoni, E., Int. J. Adhes. Adhes. 53, 57-64 (2013).

38 17. Chaboche, J. L., Girard, R., and Schaff, A., Comput. Mech. 20, 3-11 (1997). 
2 18. Chaboche, J. L., Girard, R., and Levasseur, P., Int J Damage Mech 6, 220-257 (1997).
3 .

4 19. Raous, M., Cangémi, L., and Cocu M. A., Comput. Methods Appl. Mech. Eng. 177: 383-399 5 (1999).

20. Del Piero, G., and Raous, M., Eur. J. Mech. - A/Solids 29, 496-507 (2010).

21. Alfano, G., and Sacco, E., Int. J. Numer. Methods. Eng. 68, 542-582 (2006).

22. Alfano, G., Marfia, S., and Sacco, E., Comput. Methods Appl. Mech. Eng. 196, 192-209 (2006).

23. Oinonen, A., and Marquis, G., Int. J. Adhes. Adhes. 31, 550-558 (2011).

24. Oinonen, A., and Marquis, G., Eng. Fract. Mech., 78, 163-174 (2011).

25. Halling, J., Introduction to Tribology, (Wykeham Publications, London, 1976).

26. Incremental Encoders TDS, www.hengstler.de (accessed 28 April 2015).

27. National Intstruments P. www.ni.com/products/, www.ni.com/products/.

22

28. Instruments N. Labview software, www.ni.com/labview/ (accessed 19 January 2015).

29. Henkel. Loctite ${ }^{\circledR} 243^{\mathrm{TM}}$, Technical Data Sheet, http://tds.loctite.com/tds5/docs/243 NEWEN.PDF (2010).

30. Henkel. Loctite ® 638, Technical Data Sheet. 80-82, http://tds.loctite.com/tds5/docs/638-

31. Jenoptik Group HOMMEL TESTER T500, Technical Data Sheet, http://www.agiltechnologies.com/T500_521860_e.pdf (2005).

32. Montgomery, D. C., Design and analysis of experiments, (John Wiley and Sons, New York, 2001) 5th ed. 


\section{FIGURES AND TABLES}

2

3

4

5

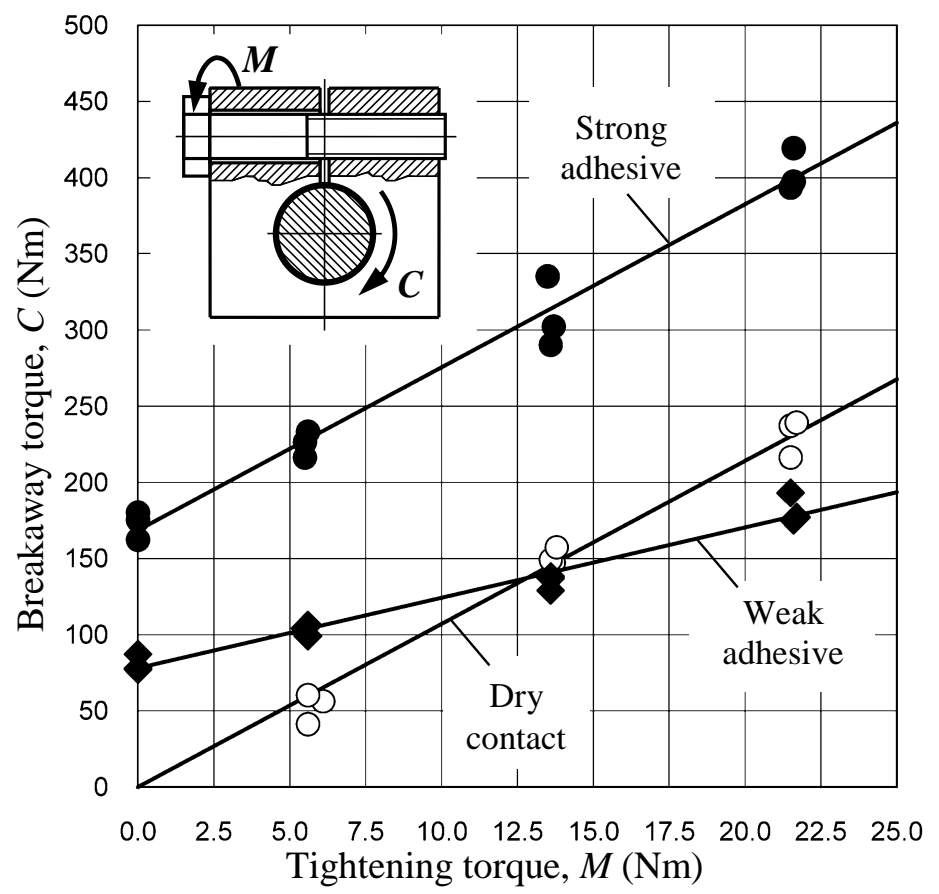

6 Figure 1: static strength has a function of clamping force in cylindrical fits, under different interface 


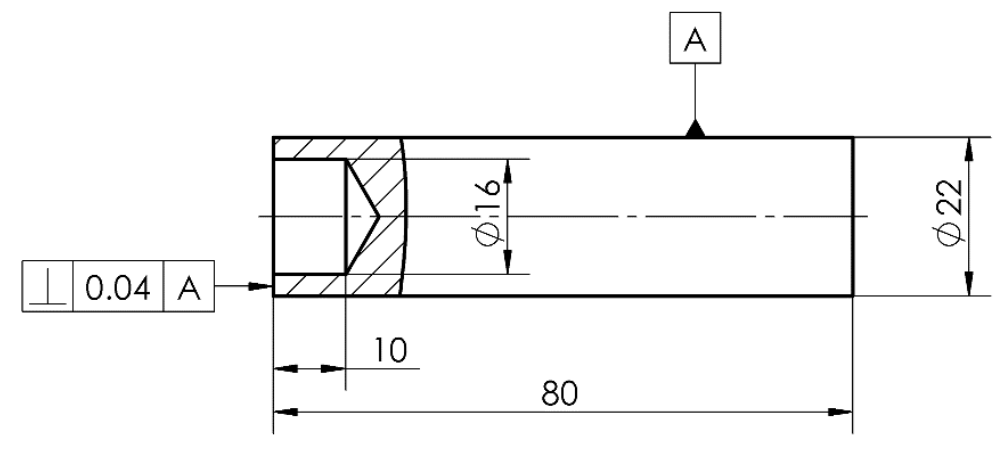

(a)

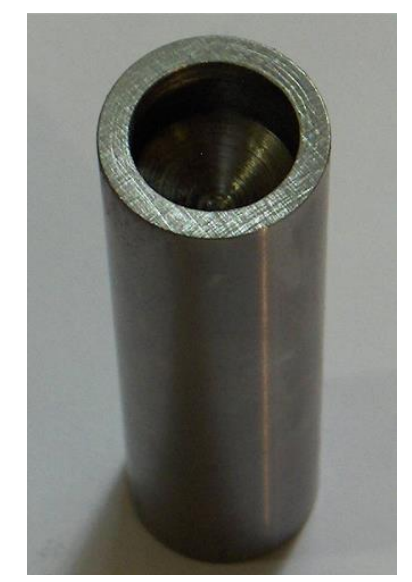

(b)

Figure 2: technical drawing of the adherend used in the experimental tests (a), and picture of the adherend manufactured by turning (b). All dimensions in millimeters. [D. Castagnetti \& E. Dragoni (2013): Experimental Assessment of a Micro-Mechanical Model for the Static Strength of Hybrid Friction-Bonded Interfaces, The Journal of Adhesion, 89:8, 642-659] 


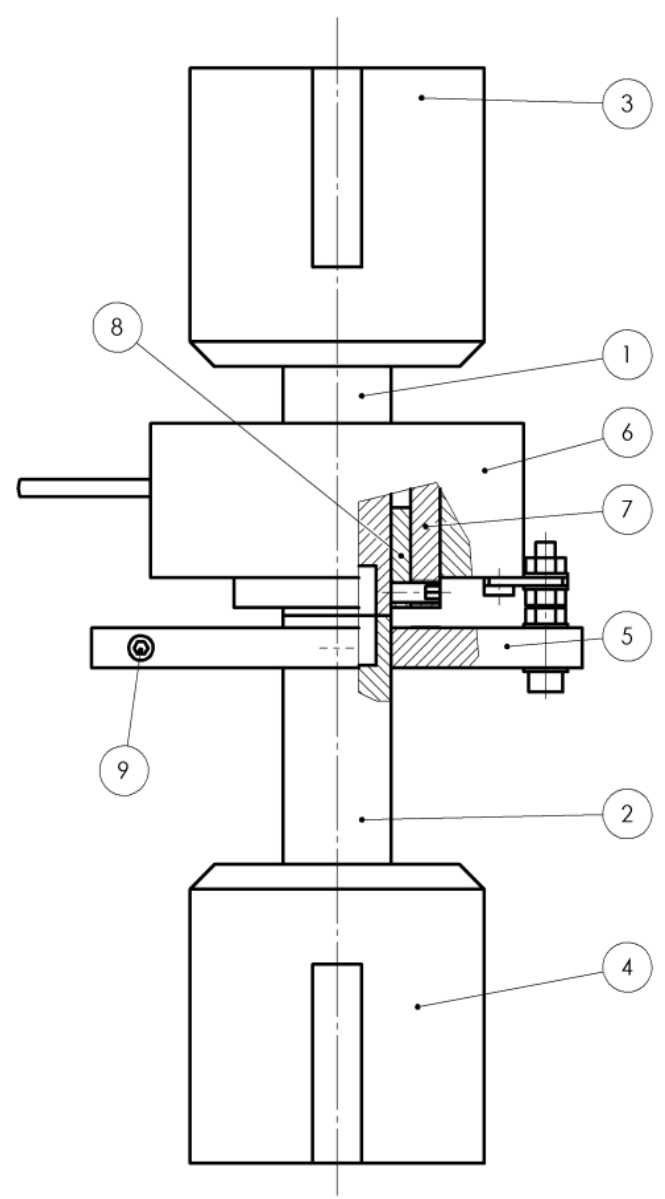

\begin{tabular}{|c|c|}
\hline$(1)$ & Top adherend \\
\hline$(2)$ & Bottom adherend \\
\hline$(3)$ & Top crosshead \\
\hline$(4)$ & Bottom crosshead \\
\hline$(5)$ & Clamp \\
\hline$(6)$ & Encoder frame \\
\hline$(7)$ & Encoder rotor \\
\hline$(8)$ & Centering bushing \\
\hline$(9)$ & Bolt-nut fastener \\
\hline
\end{tabular}

$5 \quad$ Figure 3: overall view of the specimen installed on the test machine, including the twist angle measurement system 


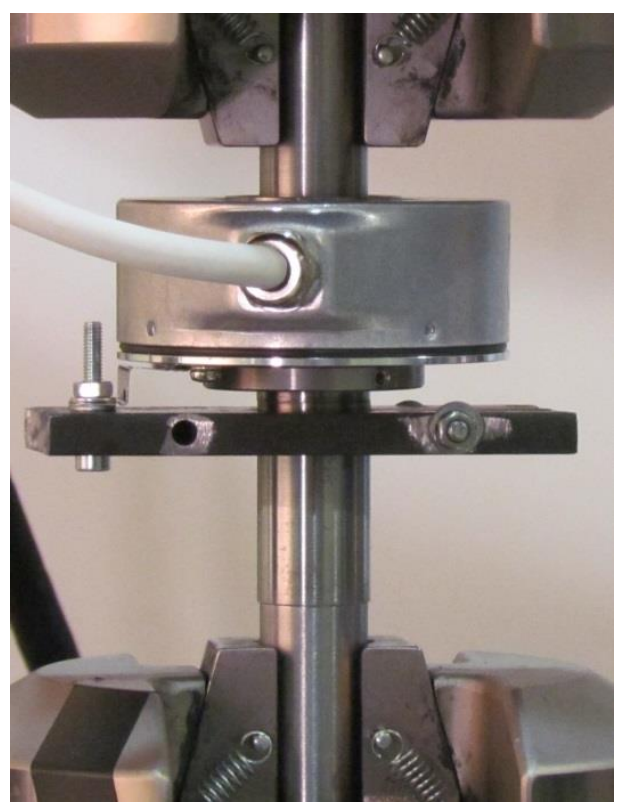

8 Figure 4: picture of the specimen fixed to the testing machine, including the twist angle measurement 9 system.

10

11 


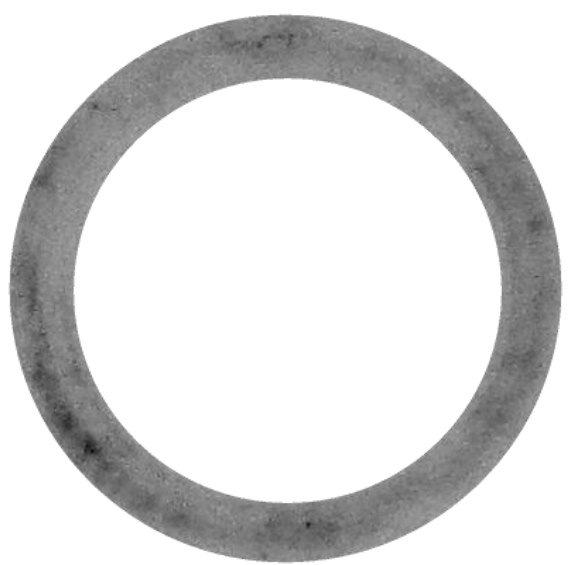

(a)

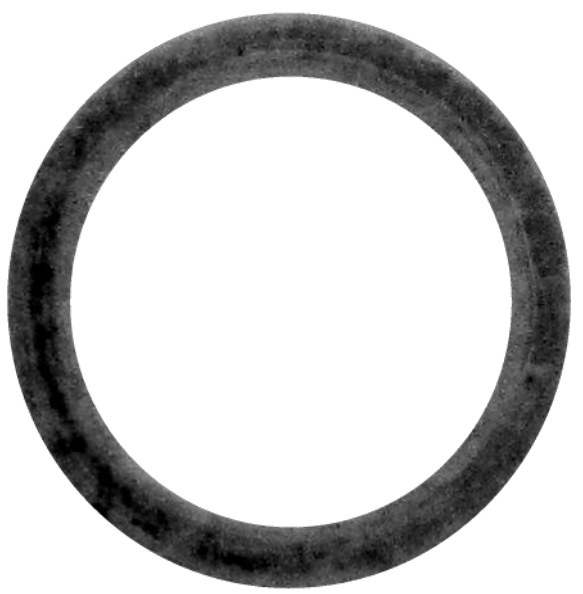

(b)

4
5

Figure 5: contact area for a specimen subject to a clamping pressure equal to $0.5 \mathrm{MPa}(\mathrm{a})$, and 6 $134 \mathrm{MPa}$ (b).

7

8 


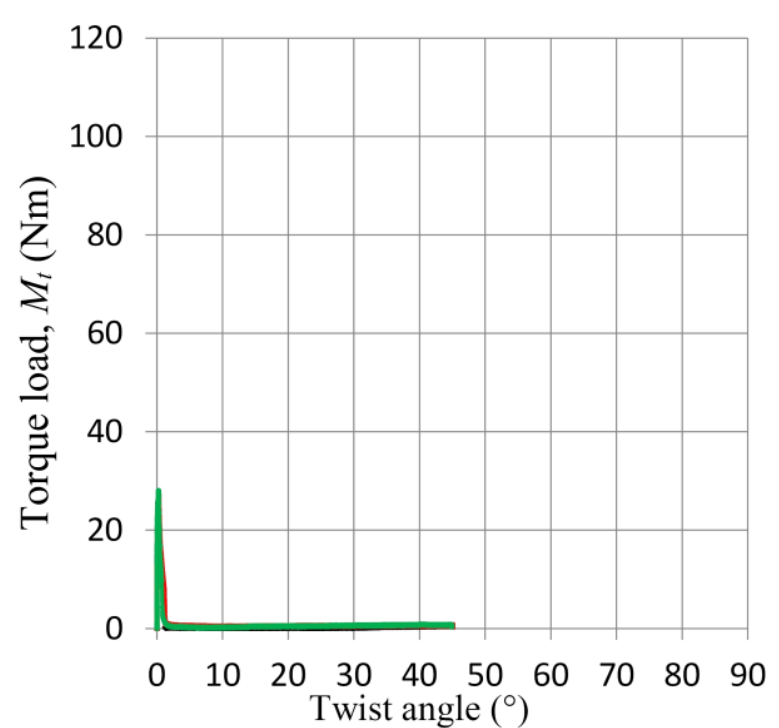

(a)

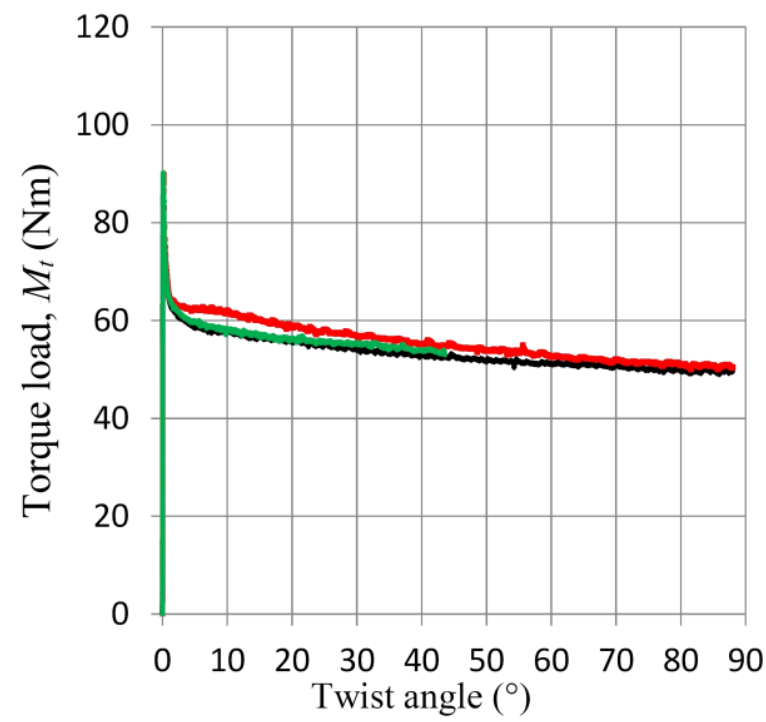

(c)

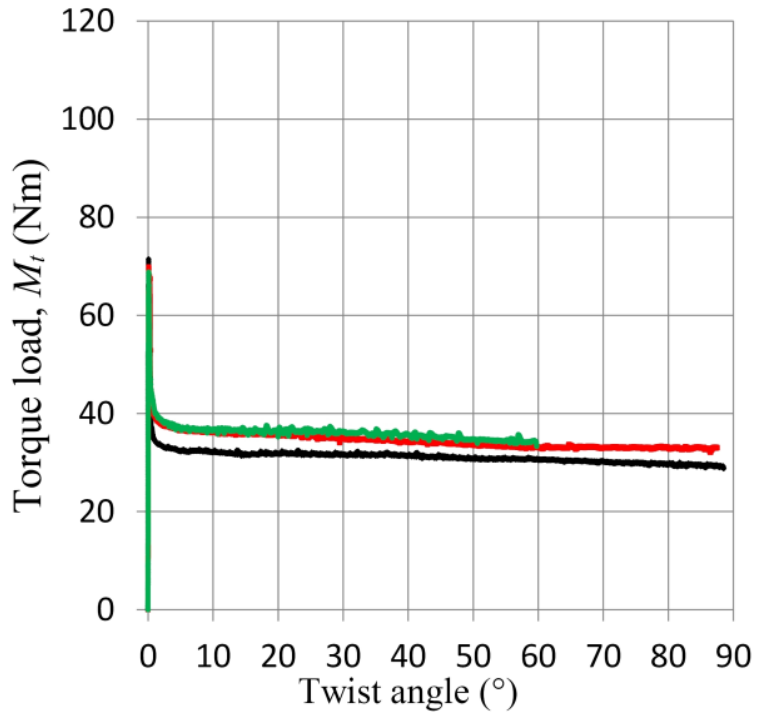

(b)

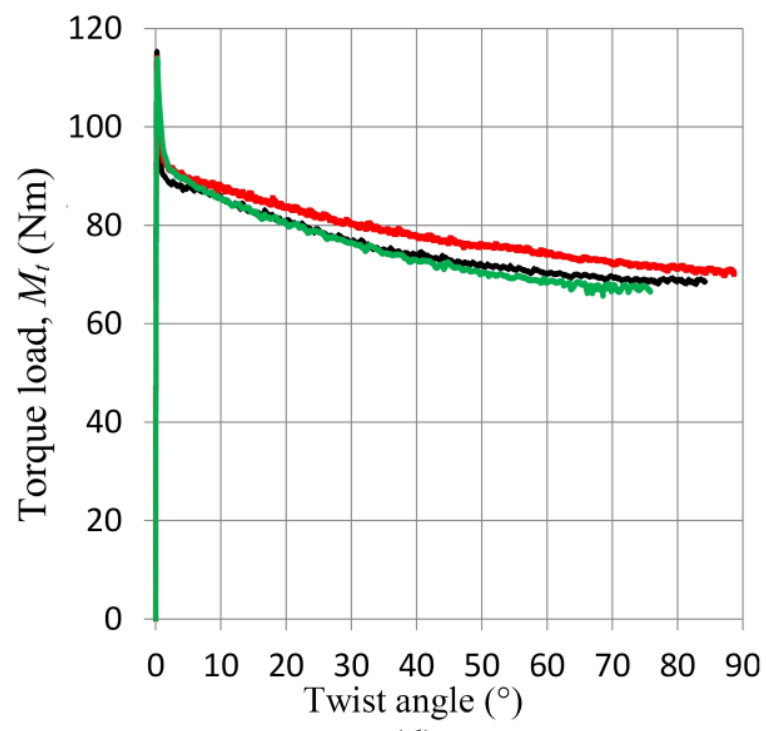

(d)

$4 \quad$ Figure 6: torque load $v s$ twist angle experimental curves for Loctite 243 anaerobic adhesive (three replicates): 0.5 $\mathrm{MPa}$ (a), $45 \mathrm{MPa}$ (b), $90 \mathrm{MPa}(\mathrm{c})$, and $134 \mathrm{MPa}$ (d) clamping pressure value. 


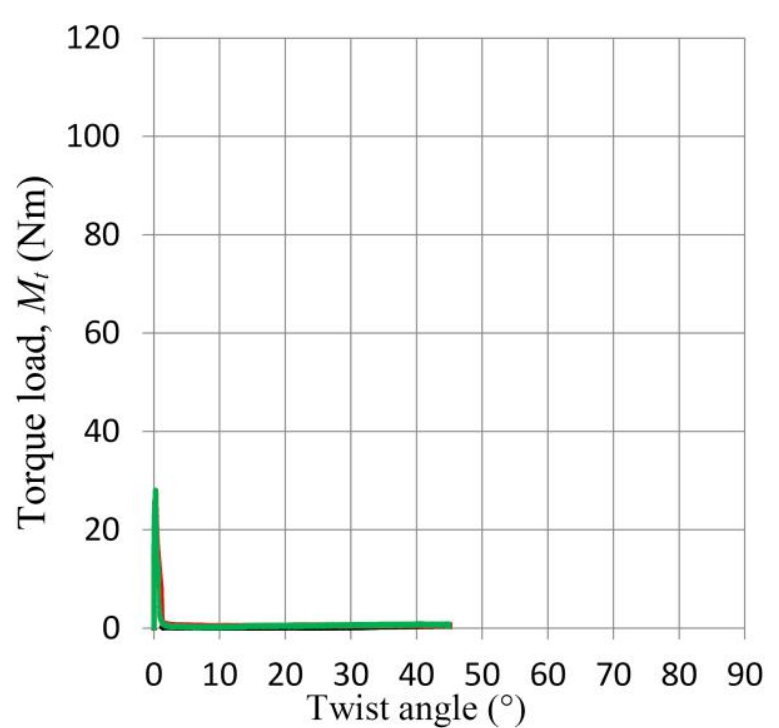

(a)

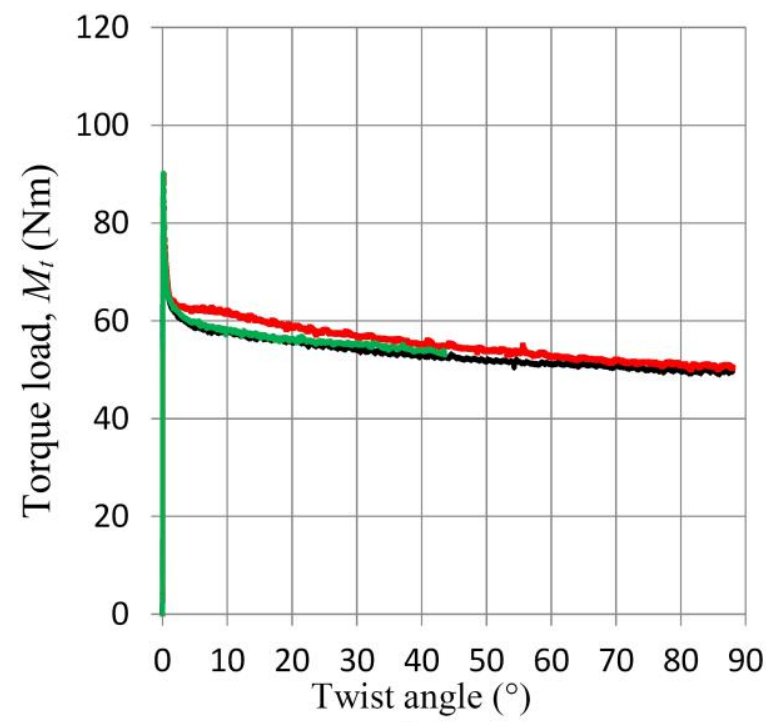

(c)

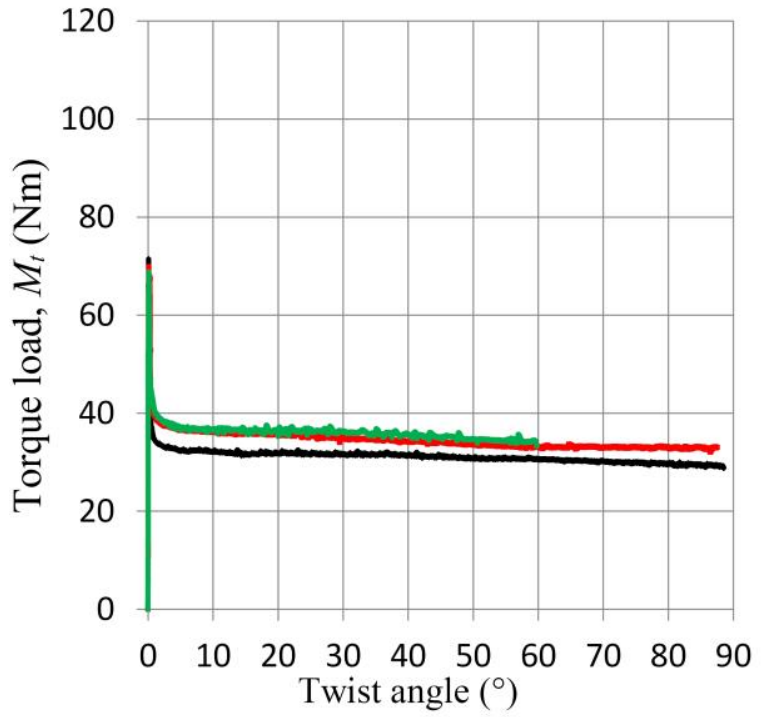

(b)

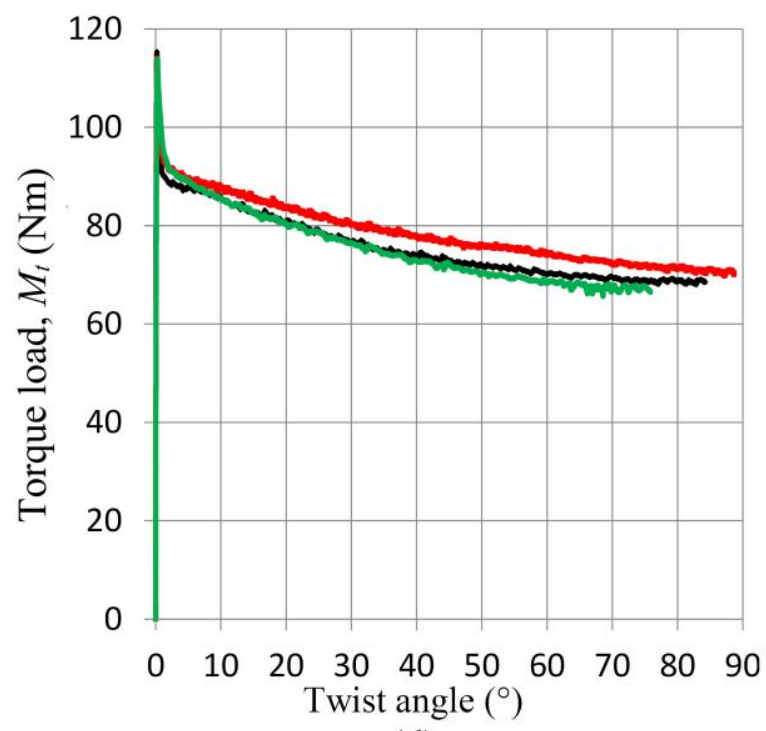

(d)

$4 \quad$ Figure 7: torque load $v s$ twist angle experimental curves for Loctite 638 anaerobic adhesive (three replicates): 0.5 $\mathrm{MPa}$ (a), $45 \mathrm{MPa}$ (b), $90 \mathrm{MPa}(\mathrm{c})$, and $134 \mathrm{MPa}$ (d) clamping pressure value. 


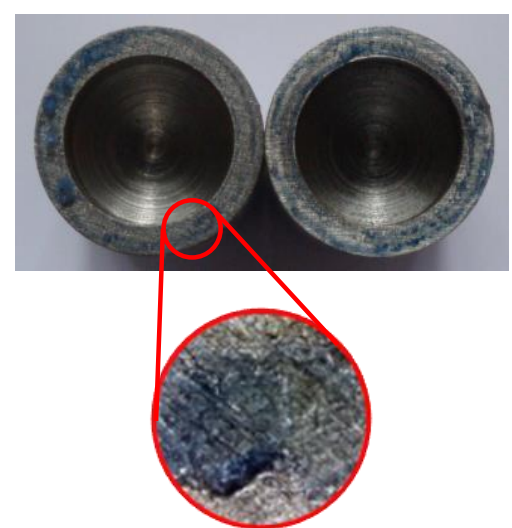

(a)

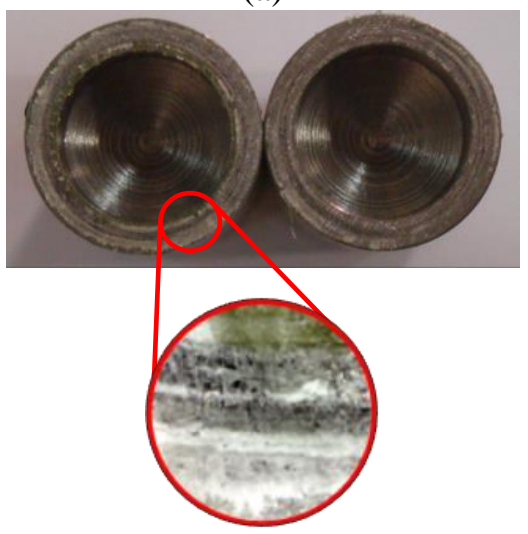

(b)

$5 \quad$ Figure 8: pictures of failed hybrid interfaces with close-up view (100 x): Loctite 243 (a), and Loctite 6638 (b) anaerobic adhesive. [D. Castagnetti \& E. Dragoni (2013): Experimental Assessment of a 7 Micro-Mechanical Model for the Static Strength of Hybrid Friction-Bonded Interfaces, The Journal of 8 Adhesion, 89:8, 642-659]

9

10 


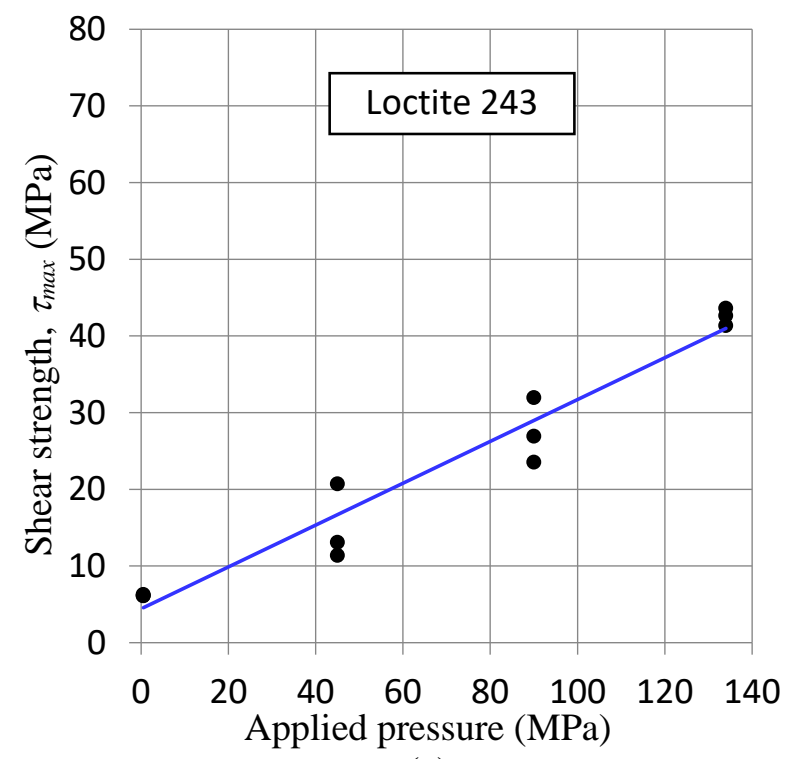

(a)

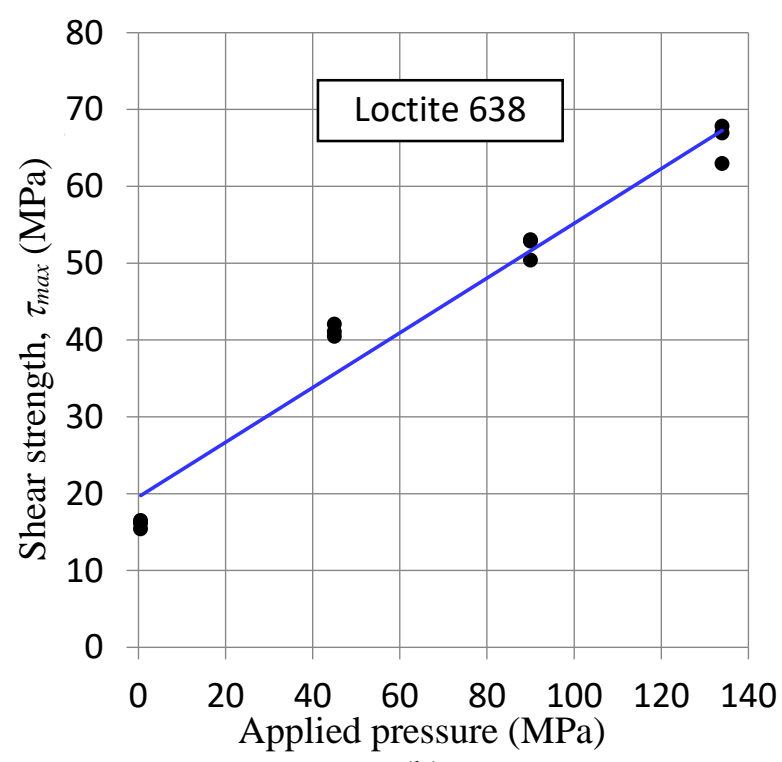

(b)

Figure 9: shear strength $\tau_{R}$ for Loctite 243 (a), and Loctite 638 (b) anaerobic adhesive.

4

5

6 


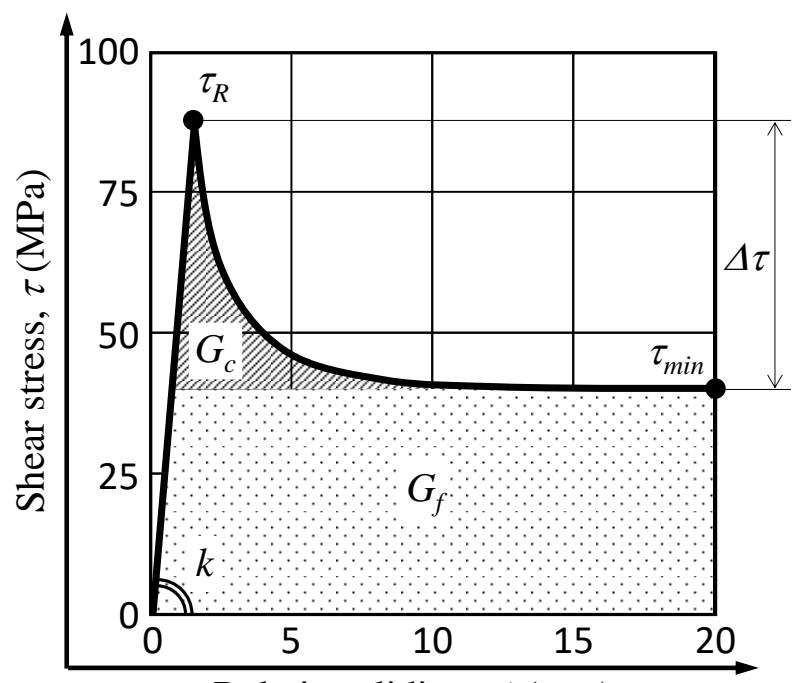

Relative sliding, $\Delta(\mathrm{mm})$

(a)

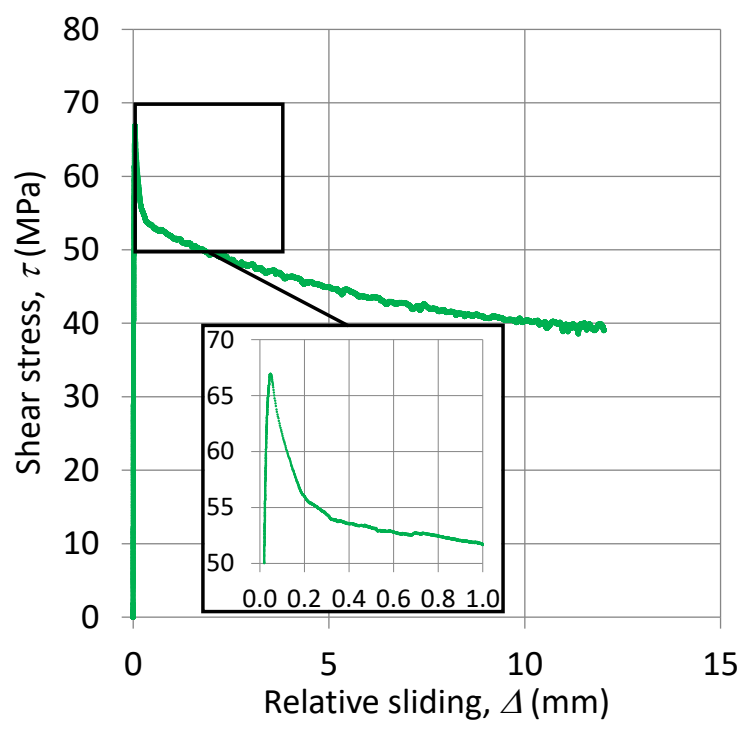

(b)

3 Figure 10: qualitative curve of the shear stress $\tau$ as a function of the relative sliding between the

4 surfaces of the hybrid interface: (a) schematic representation, (b) experimental curve for Loctite 638 at a contact pressure equal to $134 \mathrm{MPa}$, with a close-up view showing the resolution of the data.

6 


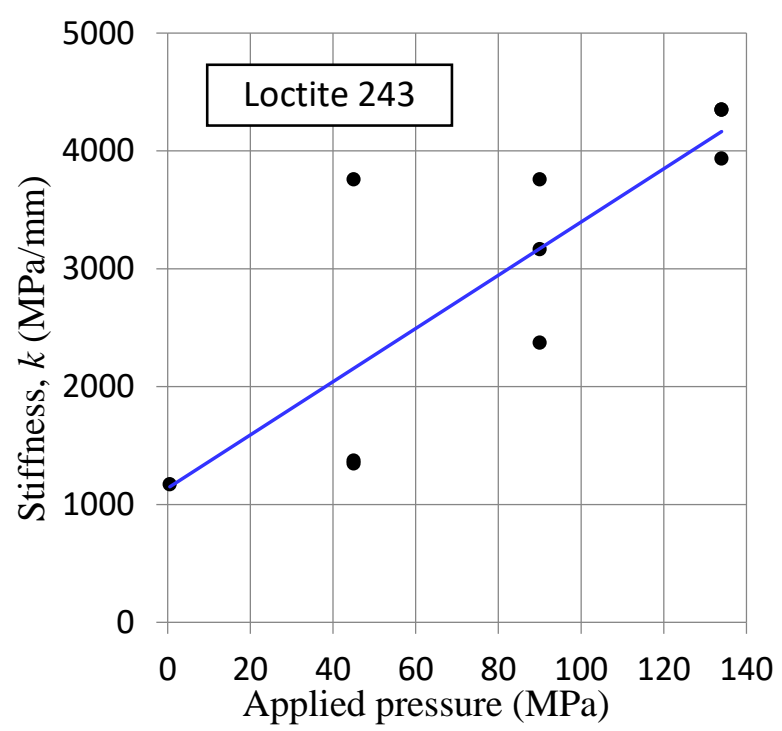

(a)

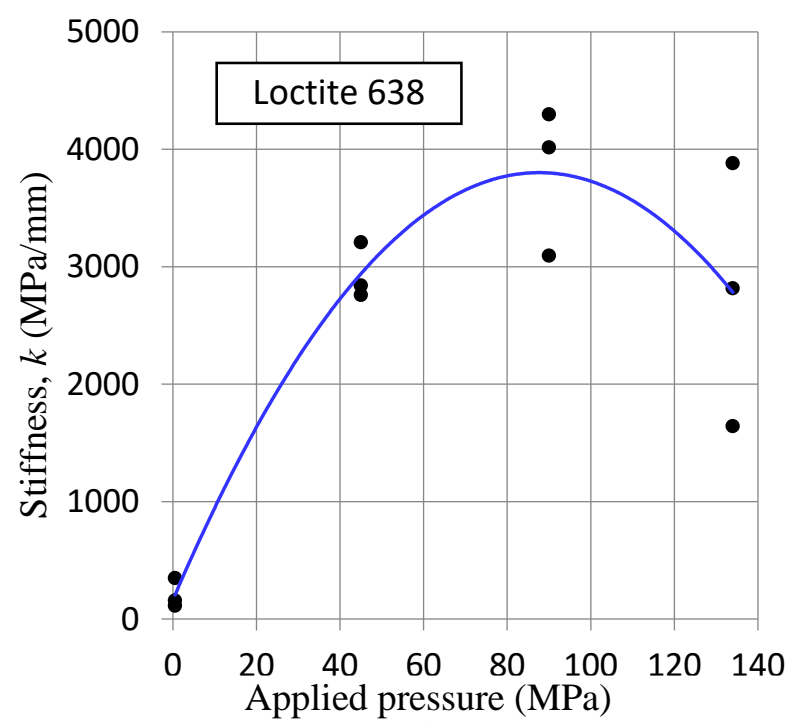

(b)

3

4

5

6

7

8 


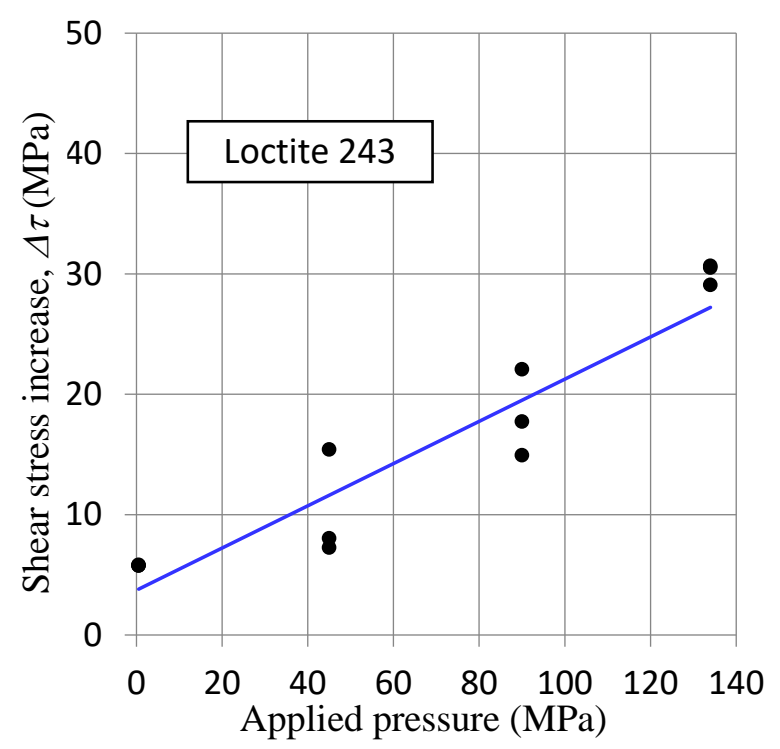

(a)

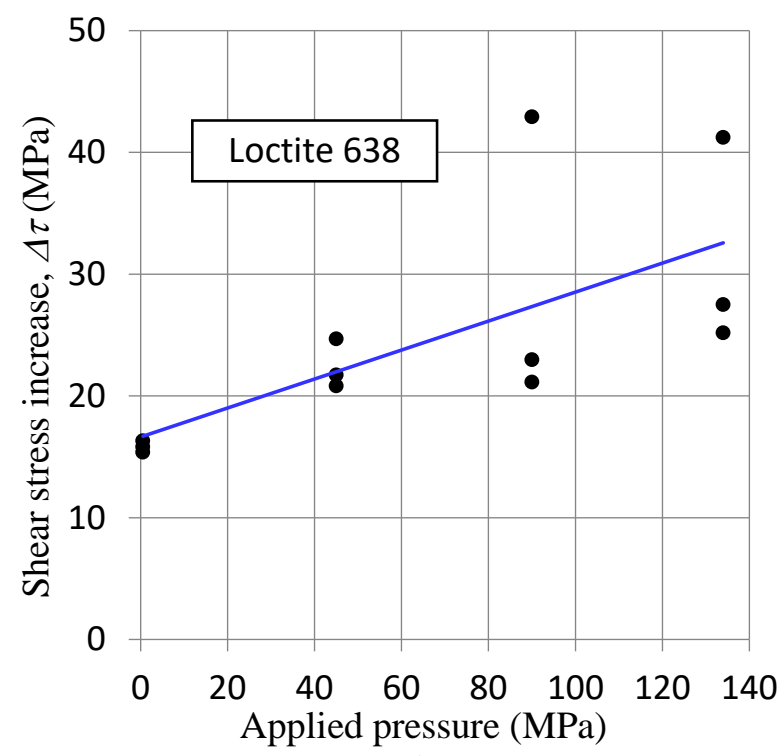

(b)

3

Figure 12: shear stress increase, $\Delta \tau$, for Loctite 243 (a), and Loctite 638 (b) anaerobic adhesive.

5

6 


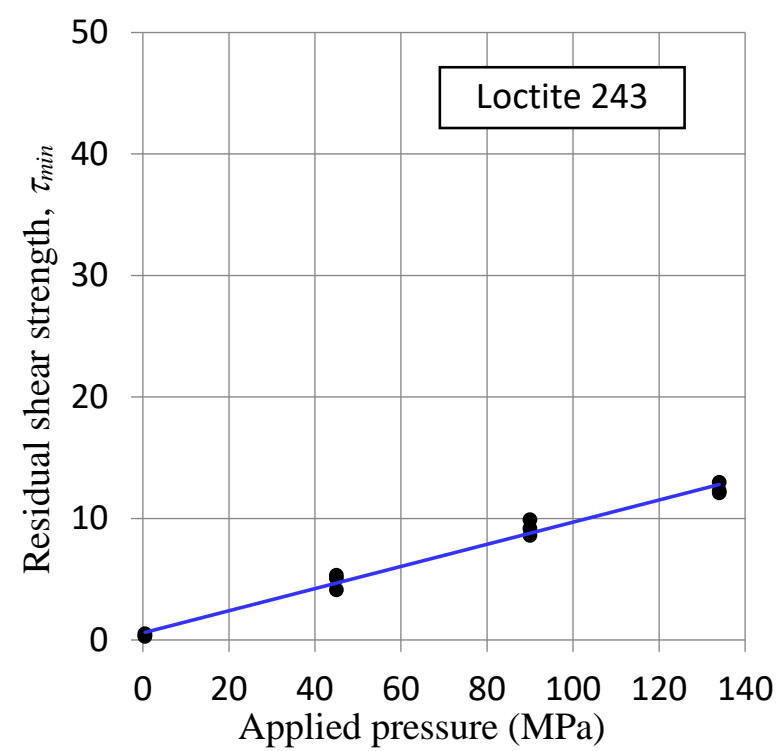

(a)

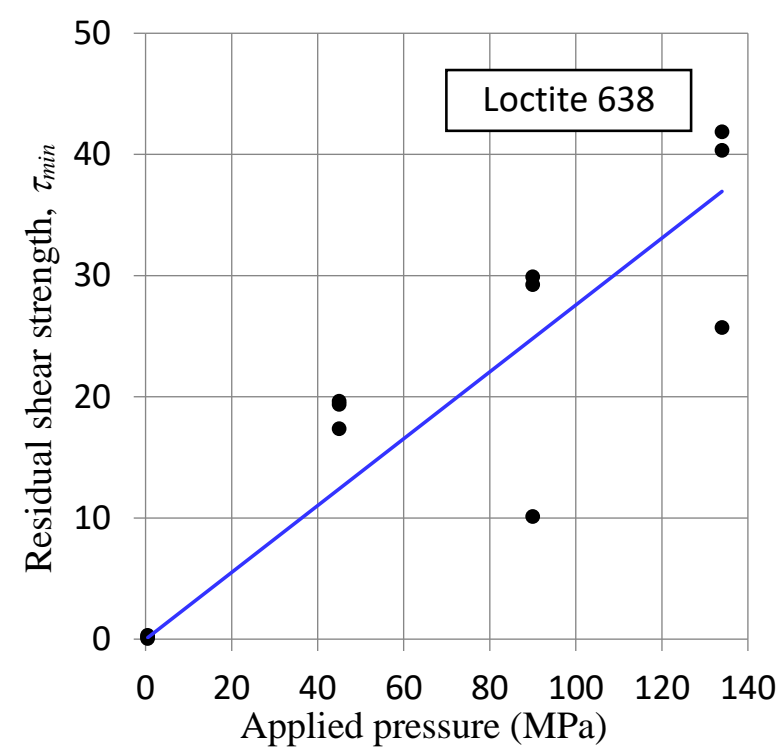

(b)

Figure 13: residual shear strength, $\tau_{\min }$, for Loctite 243 (a), and Loctite 638 (b) anaerobic adhesive.

5

6 


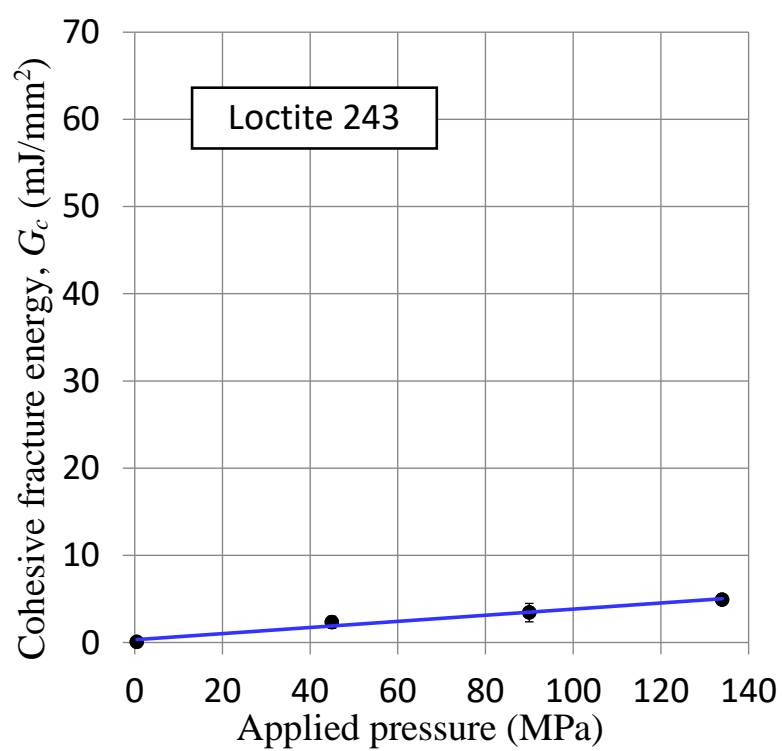

(a)

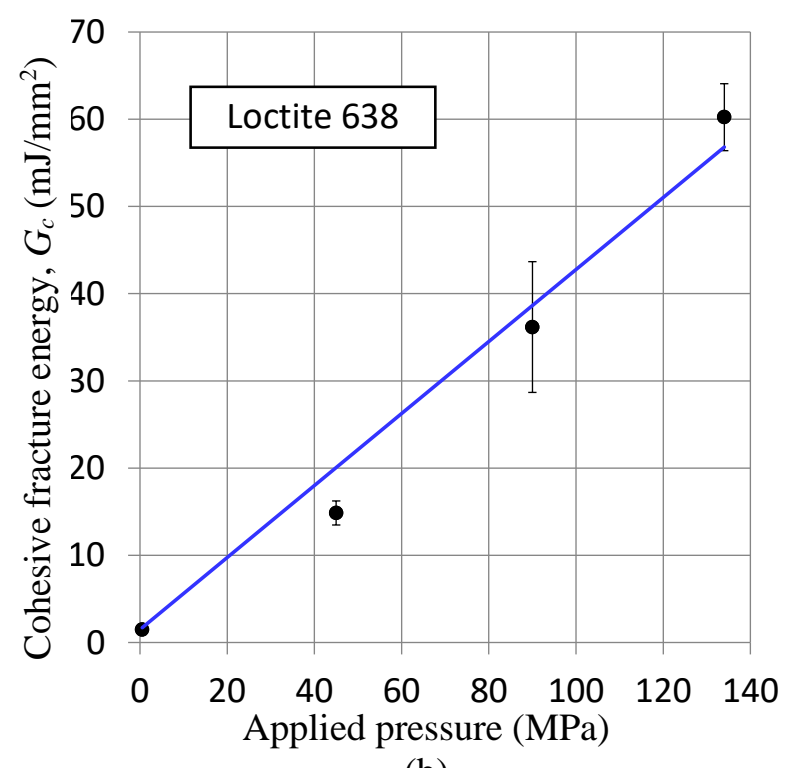

(b)

$4 \quad$ Figure 14: cohesive fracture energy, $G_{c}$, for Loctite 243 (a), and Loctite 638 (b) anaerobic adhesive. 5 


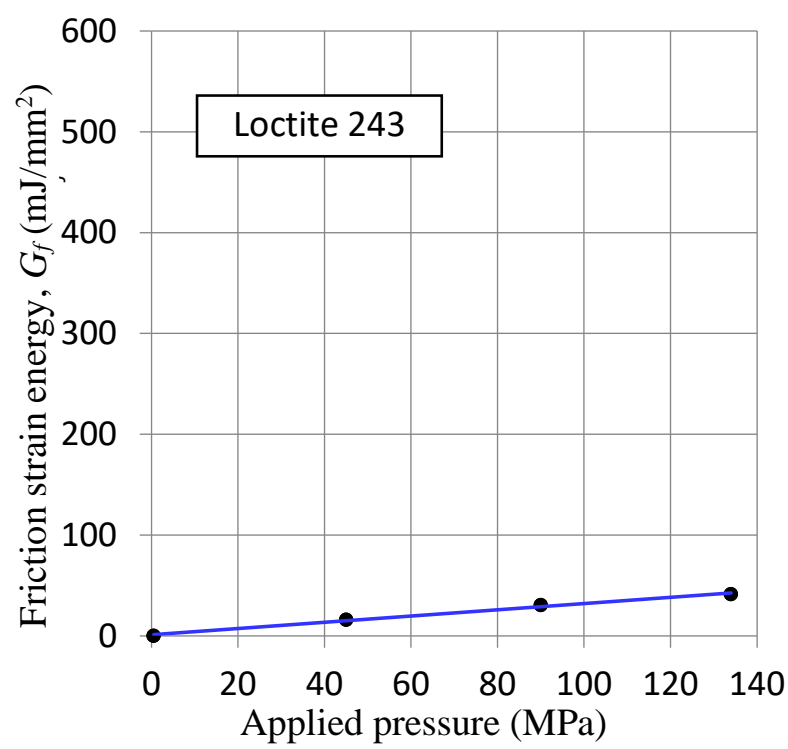

(a)

3

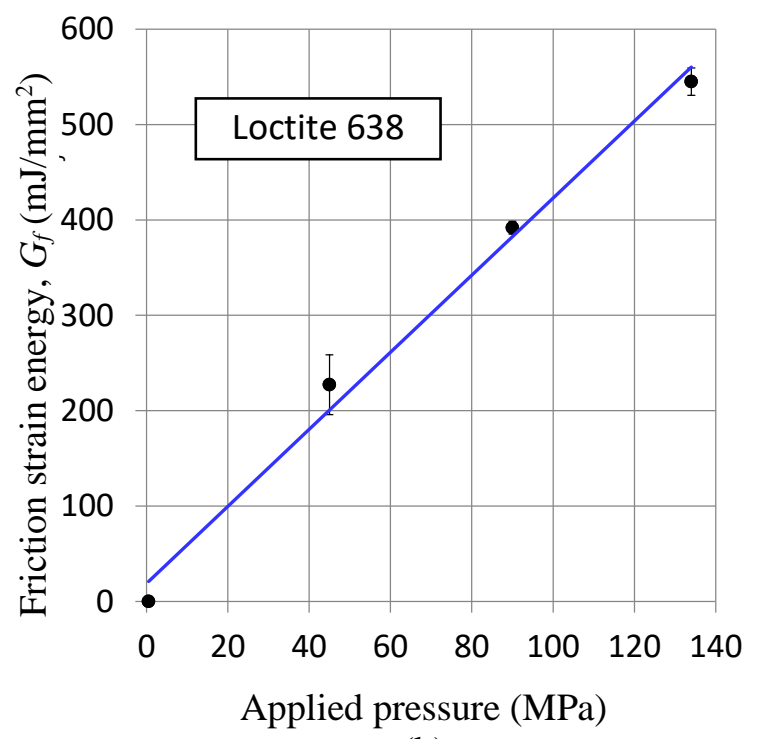

(b)

$4 \quad$ Figure 15: friction strain energy, $G_{f}$, for Loctite 243 (a), and Loctite 638 (b) anaerobic adhesive. 
Table 1: variables of the experimental test plan

\begin{tabular}{|l|c|c|c|c|}
\hline \multicolumn{1}{|c|}{ Variables } & \multicolumn{2}{c|}{-} & \multicolumn{2}{c|}{+} \\
\hline Adhesive & Loctite 243 & \multicolumn{2}{c|}{ Loctite 638 } \\
\hline Nominal contact pressure, $p(\mathrm{MPa})$ & 0.5 & 45 & 90 & 134 \\
\hline
\end{tabular}

6

7

8

9

10 Table 2: analytical relationships for the fundamental parameters of the shear stress $v s$ relative displacement curves (contact pressure $p$ in $\mathrm{MPa}$ )

\begin{tabular}{|l|l|l|}
\hline Response & Loctite 243 & Loctite 638 \\
\hline Stiffness, $k(\mathrm{MPa} / \mathrm{mm})$ & $k=22.6 p+1138.8$ & $k=-0.5 p^{2}+83.0 p+163.0$ \\
\hline Failure shear stress, $\tau_{R}$ & $\tau_{R}=0.3 p+6.1$ & $\tau_{R}=0.4 p+16.0$ \\
\hline Shear stress increase, $\Delta \tau(\mathrm{MPa})$ & $\Delta \tau=0.2 p+5.8$ & $\Delta \tau=0.1 p+15.8$ \\
\hline Residual shear strength, $\tau_{\min }$ & $\tau_{\min }=0.1 p+0.6$ & $\tau_{\min }=0.3 p+0.2$ \\
\hline Cohesive fracture energy, $G_{c}\left(\mathrm{~mJ} / \mathrm{mm}^{2}\right)$ & $G_{c}=0.3 p+0.2$ & $G_{c}=0.4 p+1.5$ \\
\hline Friction strain energy, $G_{f}\left(\mathrm{~mJ} / \mathrm{mm}^{2}\right)$ & $G_{f}=0.3 p+1.2$ & $G_{f}=4.2 p+0.1$ \\
\hline
\end{tabular}

12

13

14 